\title{
Metabolic Response of Adult Male Offspring Rats to Prenatal Caffeine Exposure
}

Ioanna Mastroleon ${ }^{1}$, Laskarina-Maria Korou ${ }^{1}$, Vasilios Pergialiotis ${ }^{1}$, Ioannis S. Vlachos ${ }^{1}$, Helen Sarlanis ${ }^{2}$ , Panagiotis Konstantopoulos ${ }^{1}$, Emmanouil Pikoulis ${ }^{3,4}$, Despina N. Perrea ${ }^{1}$, Nikolaos Kavantzas ${ }^{5}$

1. Laboratory of Experimental Surgery and Surgical Research “N.S. Christeas” (LESSR), National \& Kapodistrian University of Athens, Athens, GRC 2. First Department of Pathology, National \& Kapodistrian University of Athens, Athens, GRC 3. Third Department of Surgery, National \& Kapodistrian University of Athens, Athens, GRC 4. Surgery, Attikon University Hospital, Athens, GRC 5. Pathology, National and Kapodistrian University of Athens, Athens, GRC

Corresponding author: Ioanna Mastroleon, mastroleonio@gmail.com

\begin{abstract}
Caffeine is the most widely consumed psychoactive substance, with recommendations from health associations and regulatory bodies for limiting caffeine consumption during pregnancy being increasingly common. Prenatal exposure to caffeine has been shown to increase the risk of developing abnormalities in lipid metabolism in adult life. We further investigated the effect of prenatal caffeine exposure (PCE) (20 $\mathrm{mg} / \mathrm{kg}$ of body weight) on the metabolic "reserve" of male Sprague Dawley offspring fed on a high fructose diet in adult life. Male adult PCE offspring were assigned to four groups; Nw and Nf: offspring of control mothers (N group of mothers), having received tap water or high fructose water respectively; $\mathrm{Cw}$ and $\mathrm{Cf}$ : offspring exposed to caffeine during gestation (C group of mothers) and receiving tap water or a high fructose water solution, respectively.
\end{abstract}

Cf rats presented increased serum triglyceride level, as well as raised systolic and diastolic blood pressure levels, together with extensive renal tissue oedema in adulthood, compared to the other groups ( $\mathrm{p}<0.05$ for all comparisons). These findings show further evidence for potential detrimental metabolic effects of prenatal caffeine exposure during adulthood in this animal model.

Received 12/12/2019 Review began 12/15/2019 Review ended 02/08/2020 Published 02/16/2020

\section{(c) Copyright 2020}

Mastroleon et al. This is an open access article distributed under the terms of the Creative Commons Attribution License CC-BY 4.0., which permits unrestricted use, distribution, and reproduction in any medium, provided the original author and source are credited.
Categories: Endocrinology/Diabetes/Metabolism, Obstetrics/Gynecology, Anatomy

Keywords: caffeine, gestation, rat, lipids, blood pressure

\section{Introduction}

Caffeine is one of the most widely consumed psychoactive substances throughout the world. The daily consumption of caffeine in the US is estimated to be $211 \mathrm{mg}$ per person [1]. Caffeine readily transcends the placenta barrier and is detected in fetal circulation [2]. During pregnancy, the half-life of caffeine is, on average, $8.3 \mathrm{~h}$ longer and maybe as much as $16 \mathrm{~h}$ longer than usual, while in newborns is estimated to be between $65 \mathrm{~h}$ and $130 \mathrm{~h} \mathrm{[3].} \mathrm{However,} \mathrm{pregnant} \mathrm{women} \mathrm{are} \mathrm{usually} \mathrm{permitted} \mathrm{to} \mathrm{consume} \mathrm{a} \mathrm{moderate} \mathrm{dose} \mathrm{of}$ caffeine (<200 mg/d), as it does not appear to increase the risk of miscarriage, congenital malformations, or affect the fetal growth rate [4].

Caffeine has been reported to have anti-obesity and positive metabolic effects when consumed in adulthood [5]. Animal studies have shown that oral administration of caffeine (5 mg/kg of body weight) can increase lipolysis via catecholamine release, and rats fed on a diet with $0.05 \%$ caffeine for three or four weeks showed reduced body fat percentage [6,7]. Smaller doses of caffeine $(0.005 \%$ of food or $0.5 \mathrm{~g} / \mathrm{kg}$ of food) for eight weeks decreased body fat and systolic blood pressure and improved glucose tolerance and insulin sensitivity after a high-carbohydrate and high-fat diet [8]. Moreover, caffeine intake (1 g/L) for 15 days restored insulin sensitivity and reversed hyperglycemia and hypertension in rats, which consumed a high sucrose diet [9].

On the other hand, the interaction between antenatal caffeine exposure and fetal metabolism remains relatively unknown. A prospective cohort study linked maternal caffeine intake to an $87 \%$ increased risk for obesity in the offspring's adult life [10]. Adult rats prenatally exposed to caffeine (120 mg/kg of body weight) from gestational day 11 , which underwent a high-fat diet in adulthood, displayed higher serum glucose levels compared to non-exposed animals [11]. Additionally, in another study, serum insulin levels were lower in adult offspring of female rats exposed to caffeine during gestation and lactation $(1 \mathrm{mg} / \mathrm{mL})$, compared to control animals [12]. A recent study showed that prenatal exposure to $120 \mathrm{mg} / \mathrm{kg}$ of caffeine from gestational day 11 to 20, did not alter the basal blood glucose and insulin, but reduced $\beta$ cell fraction and mass while increasing increased insulin sensitivity in adult offspring [13]. Finally, prenatal caffeine exposure (PCE) in rats $(120 \mathrm{mg} / \mathrm{kg})$ from gestational day 11 until delivery increased the serum levels of total cholesterol and low-density lipoprotein-cholesterol (LDL-C) and decreased the high-density lipoprotein-cholesterol (HDLC) levels [14].

Currently, there are multiple indications that antenatal caffeine exposure has a differential impact on the 


\section{Blood sample analyses}

Blood samples of male offspring were collected upon adulthood (T1), one month later (T2), and two months (T3). All sample collections were performed at 9:00 am, following a 12-hour fasting period, using capillary tubes introduced into the medial retro-orbital venous plexus under light ether anaesthesia. Serum was separated by centrifugation at $3000 \mathrm{rpm}$ for $10 \mathrm{~min}$ and was stored at $-80^{\circ} \mathrm{C}$ awaiting analysis.

Serum concentrations of total cholesterol and triglycerides were determined using an enzymatic phenolaminophenazone (PAP) commercial kit ("Biosis" - Biotechnological Applications, Athens, Greece), whereas HDL-cholesterol was determined with a cholesterol enzymatic photometric method. LDL-cholesterol was determined using the Friedewald formula. Serum glucose levels were measured by colorimetric method.

\section{Blood pressure measurements}

Systolic and diastolic blood pressure was recorded by using a non-invasive tail-cuff blood pressure system (Coda 2, Kent Scientific, U.S.A.) at one month, following the high fructose feeding between 10 am and $2 \mathrm{pm}$. The animals were familiarized with the blood pressure measuring equipment before initiation of the measurements. For each rat, three blood pressure measurements were recorded, and their average was registered as their blood pressure measurement.

\section{Glucose tolerance test (GTT)}

The glucose tolerance test was performed one week before euthanasia. After a 16h, overnight fasting period, blood samples were collected from the dorsal pedal vein of the rats, and fasting serum glucose levels were determined by glucometer. The animals were weighed to estimate glucose doses ( $1.5 \mathrm{~g}$ of glucose $/ \mathrm{kg}$ body weight). The rats were then injected (intraperitoneal) with the glucose solution, and subsequently, blood was collected from the dorsal pedal vein of the animals at $30 \mathrm{~min}, 60 \mathrm{~min}$, and $120 \mathrm{~min}$ to measure serum glucose levels.

\section{Histopathology analysis of renal and hepatic tissue samples}

The specimens were fixed in $10 \%$ formaldehyde for 24 hours. Paraffin-embedded sections of $4 \mu \mathrm{m}$ depth were stained with hematoxylin and eosin. All sections were examined by light microscopy at different magnifications (x50, x100).

\section{Statistical analysis}

Quantitative variables are described as a median [interquartile range]. The Kruskal-Wallis test was implemented for multiple group comparisons, whereas the Mann-Whitney U test was used for post hoc multiple testing. In all cases of multiple hypothesis testing, Benjamini-Hochberg's false discovery rate (FDR) was applied to assess differences and control family-wise errors as $<0.05$. All tests were two-sided. $\mathrm{P}<0.05$ was indicative of a statistically significant difference.

\section{Results}

No differences in litter size and sex ratio of the offspring were observed. Each litter comprised an average of 10 pups. The number of offspring was 100 in total (51 female and 49 male) for group $\mathrm{N}$ and 96 (46 female and 50 male) for group C, without a significant difference. There was a significant difference, however, in the birth weight of offspring between groups $\mathrm{N}$ and $\mathrm{C}$, with caffeine-administered mothers having lowerweight offspring ( $\mathrm{p}<0.001)$ (Group N: $55 \mathrm{~g}(50-64 \mathrm{~g})$, Group C: $46 \mathrm{~g}(44-48 \mathrm{~g}))$.

Regarding the glucose tolerance test, $\mathrm{Nf}$ and $\mathrm{Cf}$ animals had higher median glucose levels at 30', 60', and 120' of the glucose tolerance test, but the differences were not identified as statistically significant. Also, there was no significant difference in fasting glucose levels among groups at any of the time points, but there was a significant difference for the $\mathrm{Nw}, \mathrm{Nf}, \mathrm{Cw}$, and $\mathrm{Cf}$ groups in the different time points (Table 1).

\begin{tabular}{|c|c|c|c|c|c|}
\hline Variable & Nw group & Nf group & Cw group & Cf group & $\begin{array}{l}\text { p-value (Kruskal- } \\
\text { Wallis) }\end{array}$ \\
\hline GTT 0 & 100 (85-122) & 95 (71-129) & $105.5(58-131)$ & $108(61-130)$ & 0.829 \\
\hline GTT 30 & $171.5(122-281)$ & $223(176-321)$ & 215 (95-386) & 210.5 (134-352) & 0.349 \\
\hline GTT 60 & 129 (90-162) & 151.5 (114-201) & 124 (95-283) & 175.5 (100-220) & 0.089 \\
\hline GTT 120 & 111 (83-123) & 120 (84-147) & 99 (84-189) & 114.5 (84-145) & 0.337 \\
\hline Glucose T1 & $140(130-165)$ & $145(120-200)$ & $147.5(120-170)$ & 147.5 (120-170) & 0.731 \\
\hline Glucose T2 & $150(140-180)$ & 155 (120-175) & $155(125-180)$ & $157.5(145-190)$ & 0.607 \\
\hline
\end{tabular}




\section{Cureus}

\begin{tabular}{|c|c|c|c|c|c|}
\hline Glucose T3 & 177.5 (135-220) & $180(138-230)$ & $187.5(165-210)$ & $195(168-240)$ & 0.401 \\
\hline p-value (Friedman) & 0.001 & 0.007 & $<0.001$ & 0.001 & \\
\hline Total cholesterol (T 1) & $99.5(85-110)$ & $105(90-120)$ & $95(80-110)$ & $97.5(80-115)$ & 0.171 \\
\hline Total cholesterol (T2) & $82.5(70-100)$ & $82.5(70-120)$ & $82.5(80-100)$ & $80(65-90)$ & 0.473 \\
\hline Total cholesterol (T3) & $75(60-100)$ & $85(65-100)$ & $80(65-95)$ & 75 (65-95) & 0.705 \\
\hline p-value (Friedman) & 0.001 & 0.013 & 0.002 & $<0.001$ & \\
\hline Triglycerides (T1) & $63(45-95)$ & $70(50-126)$ & $77.5(60-120)$ & $80(60-140)$ & 0.254 \\
\hline Triglycerides (T2) & $87.5(65-115)^{\mathrm{a}, \mathrm{b}}$ & $112.5(60-195)^{\mathrm{a}, \mathrm{c}}$ & $82.5(45-150)^{c, d}$ & $140(90-190)^{b, d}$ & $0.002 *$ \\
\hline Triglycerides (T3) & $70(40-105)^{a, b}$ & $97.5(60-150)^{\mathrm{a}, \mathrm{c}, \mathrm{d}}$ & $65(40-80)^{c, e}$ & $150(80-240)^{b, d, e}$ & $<0.001$ * \\
\hline p-value (Friedman) & 0.053 & 0.266 & 0.020 & 0.012 & \\
\hline HDL (T1) & $53(46-61)$ & $56(48-65)$ & $51.5(45-59)$ & $53(46-60)$ & 0.284 \\
\hline HDL (T2) & $54(46-60)$ & $55.5(49-66)$ & $75(46-150)$ & $56.5(47-190)$ & 0.572 \\
\hline HDL (T3) & $54(46-60)$ & $54.5(48-65)$ & $50.5(40-58)$ & $53(46-59)$ & 0.249 \\
\hline p-value (Friedman) & 0.168 & 0.053 & 0.001 & 0.010 & \\
\hline LDL (T1) & $31(21-37)$ & $35(14-43)$ & $29(11-38)$ & $60(6-36)$ & 0.269 \\
\hline LDL (T2) & $10(5-22)^{a, b}$ & $10(1-38)$ & $17(8-31)^{a, c}$ & $5(2-17)^{b, c}$ & $0.013^{*}$ \\
\hline LDL (T3) & $10(0-21)$ & $5(2-26)$ & $16(4-22)$ & $12.5(0-24)$ & 0.328 \\
\hline p-value (Friedman) & 0.001 & 0.007 & 0.050 & 0.023 & \\
\hline Systolic blood pressure & $\begin{array}{l}135(122- \\
143)^{a, b, c}\end{array}$ & $152(148-162)^{\mathrm{a}, \mathrm{d}, \mathrm{e}}$ & $\begin{array}{l}144(140- \\
155)^{b, d, f}\end{array}$ & $\begin{array}{l}160.5(152- \\
174)^{c, e, f}\end{array}$ & $<0.001$ \\
\hline $\begin{array}{l}\text { Diastolic blood } \\
\text { pressure }\end{array}$ & $106(80-117)^{a, b}$ & $\begin{array}{l}120.5(107- \\
139)^{a, c, d}\end{array}$ & $106(88-138)^{c, e}$ & $130(112-143)^{b, d, e}$ & $<0.001$ \\
\hline Weight (T1) & $336(296-360)$ & 337 (296-378) & 317 (264-352) & $344(310-380)$ & 0.131 \\
\hline Weight (T2) & $414(378-450)$ & $400(366-458)$ & $388(348-410)$ & $403(392-456)$ & 0.148 \\
\hline Weight (T3) & $444(404-460)$ & $446(370-512)^{a}$ & $418(368-456)^{a, b}$ & $442(406-494)^{b}$ & $0.042 *$ \\
\hline
\end{tabular}

TABLE 1: Serum glucose levels $(\mathrm{mg} / \mathrm{dl})$ recorded following the glucose tolerance tests (GTTO, GTT30, GTT60, GTT120) and at T1, T2 and T3; and serum levels of total cholesterol (mg/dl), HDL cholesterol (mg/dl), LDL cholesterol ( $\mathrm{mg} / \mathrm{dl})$, triglycerides $(\mathrm{mg} / \mathrm{dl})$ and blood pressure (systolic, diastolic and mean blood pressure) levels per animal group studied ( $\mathrm{Nw}, \mathrm{Nf}, \mathrm{Cw}, \mathrm{Cf}$ ) at $\mathrm{T} 1, \mathrm{~T} 2$, and T3

Data are expressed as median (range). P-values marked with (*) show a statistically significant difference $(p<0.05)$.

Post hoc analysis to determine differences among groups for the Kruskal-Wallis analysis: Figures sharing the same superscript letters differentiate significantly from each other.

TGL (T2): 1) Nw vs Nf $P=0.025$, 2) Nw vs Cf $p=0.001$, 3) Nf vs Cw $p=0.045,4)$ Cw vs Cf $p=0.004$

TGL (T3): 1) Nw vs Nf $p=0.015,2) \mathrm{Nw}$ vs $\mathrm{Cf} p=0.001,3) \mathrm{Nf}$ vs $\mathrm{Cw} \mathrm{p}=0.002,4) \mathrm{Nf}$ vs $\mathrm{Cf} \mathrm{p}=0.037,5)$ Cw vs $\mathrm{Cf} \mathrm{p}<0.001$

LDL (T2): 1) Nw vs Cw $p=0.052,2) N w$ vs $C f p=0.015,3)$ Cw vs $C f p=0.001$

Systolic blood pressure: 1) Nw vs $N f p<0.001$, 2) Nw vs $C w p<0.001,3) N w$ vs $C f p<0.001$, 4) Nf vs $C w p=0.025,5) N f$ vs $C f p=0.019,6) C w$ vs $C f$ $\mathrm{p}<0.001$

Diastolic blood pressure: 1) Nw vs Nf $\mathrm{p}=0.002$, 2) Nw vs $\mathrm{Cf} p<0.001,3) \mathrm{Nf}$ vs $\mathrm{Cw} p=0.041$, 4) Nf vs Cf $\mathrm{p}=0.053,5) \mathrm{Cw}$ vs $\mathrm{Cf} p=0.005$

Weight (T3): 1) Nf vs Cw p=0.041, 2) Cw vs $C f p=0.005$ 


\title{
Cureus
}

\author{
Nw group - offspring of control mothers receiving tap water \\ $\mathrm{Nf}$ group - offspring of control mothers receiving high fructose water \\ $\mathrm{Cw}$ - offspring exposed to caffeine during gestation receiving tap water \\ Cf - offspring exposed to caffeine during gestation receiving high fructose water \\ GTT - glucose tolerance test; HDL - high-density lipoprotein; LDL - low-density lipoprotein
}

No significant differences were observed between the groups at $\mathrm{T} 1$ measurements regarding serum lipid levels (Table 1). At T2, animals in group Nw had lower serum triglyceride levels in comparison with animals of both Nf $(\mathrm{P}=0.025)$ and $\mathrm{Cf}(\mathrm{P}=0.001)$ groups. Triglyceride levels in group $\mathrm{Cw}$ were lower than the respective levels in $\mathrm{Nf}(\mathrm{P}=0.045)$ and $\mathrm{Cf}(\mathrm{P}=0.004)$ groups. Serum LDL cholesterol levels analysis at $\mathrm{T} 2$ revealed that animals in Cw group had increased levels in comparison with LDL cholesterol levels in group Nw $(\mathrm{P}=0.052)$ and group $\mathrm{Cf}(\mathrm{P}=0.001)$, with animals in group Nw displaying higher such levels than those in group $\mathrm{Cf}$ $(\mathrm{P}=0.015)$. At $\mathrm{T} 3$, animals in group $\mathrm{Nw}$ had lower triglyceride levels than animals in groups $\mathrm{Nf}(\mathrm{P}=0.015)$ and $\mathrm{Cf}(\mathrm{P}=0.001)$. Animals in group Nf had higher such levels than those in group $\mathrm{Cw}(\mathrm{P}=0.002)$, but lower triglyceride levels than $\mathrm{Cf}$ animals $(\mathrm{P}=0.037)$. Moreover, group $\mathrm{Cw}$ animals had lower triglyceride levels than animals of group $\mathrm{Cf}(\mathrm{P}<0.001)$.

Comparison of systolic blood pressure measurements between the groups showed that animals of groups Nw and $\mathrm{Cw}$ had a lower systolic pressure when compared to those recorded in animals of groups $\mathrm{Nf}$ (Nw vs $\mathrm{Nf}$ and $\mathrm{Cw}$ vs $\mathrm{Nf}, \mathrm{P}<0.001$ and $\mathrm{P}=0.025$ respectively) and $\mathrm{Cf}$ (Nw vs $\mathrm{Cf}, \mathrm{P}<001$; $\mathrm{Cw}$ vs $\mathrm{Cf}, \mathrm{P}<0.001$ ). Also, rats in $\mathrm{Nw}$ and $\mathrm{Nf}$ groups had lower systolic blood pressure than animals in $\mathrm{Cw}$ and Cf groups respectively (Nw vs $\mathrm{Cw}, \mathrm{P}<0,001$; Nf vs $\mathrm{Cf}, \mathrm{P}=0.019)$. Diastolic blood pressure was higher in group $\mathrm{Nf}$ in comparison to group $\mathrm{Nw}$ $(\mathrm{P}=0.002)$, and in group $\mathrm{Cf}$ compared to $\mathrm{Cw}(\mathrm{P}=0.005)$. Statistical analysis also indicated increased diastolic blood pressure measurements in group Cf compared to group $\mathrm{Nf}(\mathrm{P}=0.053)$, and lower values in group $\mathrm{Nw}$ when compared to group $\mathrm{Cf}(\mathrm{P}<0.001)$. Finally, group Nf had higher diastolic pressure than group $\mathrm{Cw}$ $(\mathrm{P}=0.041)$ (Table 1).

Significant differences in body weight were recorded at the end of the experimental period between groups. Specifically, $\mathrm{Cw}$ group had lower body weight levels compared to $\mathrm{Nf}(\mathrm{P}=0.041)$ and $\mathrm{Cf}(\mathrm{P}=0.005)$ groups. However, there were no differences identified between the two control offspring group (Nw, Cw). Fructosediet groups had significantly less food consumption vs control diet groups, since they received extra calories from the fructose added in the water. At $\mathrm{T} 2$ and $\mathrm{T} 3$, Nf and $\mathrm{Cf}$ animals increased water consumption compared to T1, leading to receiving higher amounts of fructose during the final month of the experiment. Importantly, no differences in food and water consumption were identified between the two control diet groups (Nw vs $\mathrm{Cw}$ ) or between the two fructose diet groups (Nf vs Cf) (Table 2). 


\section{Cureus}

\begin{tabular}{|l|llllll|}
\hline & & Nw & Nf & Cw & Cf \\
\hline Food Consumption (g/day) & T1 & $31(11)^{\mathrm{a}}$ & $31(18.2)$ & $28(5)^{\mathrm{b}}$ & $15(3.2)^{\mathrm{a}, \mathrm{b}}$ \\
& T2 & $27(2)^{\mathrm{a}, \mathrm{b}}$ & $26(10.5)^{\mathrm{a}}$ & $26(2.25)^{\mathrm{c}}$ & $16(6)^{\mathrm{b}, \mathrm{c}}$ \\
& T3 & $27(2.75)^{\mathrm{a}, \mathrm{b}}$ & $14(5.25)^{\mathrm{a}}$ & $25(2)^{\mathrm{c}}$ & $14.5(1.5)^{\mathrm{b}, \mathrm{c}}$ \\
Water Intake (ml/day) & T1 & $34.5(5.75)^{\mathrm{a}}$ & $52(25.25)^{\mathrm{a}, \mathrm{b}}$ & $34.5(1.5)^{\mathrm{b}, \mathrm{c}}$ & $57(8.25)^{\mathrm{c}}$ \\
& T2 & $34(4.75)^{\mathrm{a}, \mathrm{b}}$ & $85(21.75)^{\mathrm{a}, \mathrm{c}}$ & $33(6.75)^{\mathrm{c}, \mathrm{d}}$ & $84(6.5)^{\mathrm{b}, \mathrm{d}}$ \\
& T3 & $34(1.75)^{\mathrm{a}, \mathrm{b}}$ & $84(18)^{\mathrm{a}, \mathrm{c}}$ & $35.5(6.25)^{\mathrm{c}, \mathrm{d}}$ & $84(18.5)^{\mathrm{b}, \mathrm{d}}$ \\
\hline
\end{tabular}

TABLE 2: Food consumption (g/day) and water intake (ml/day) per animal group studied at T1, T2, and T3

Data are expressed as median (interquartile range).

Post hoc analysis to determine differences among groups for the Kruskal-Wallis analysis: Figures sharing the same superscript letters differentiate significantly from each other.

Food Consumption

T1: Nw vs $C f, P=0.002 ; C w$ vs $C f, P<0.001$

T2: Nw vs Nf, $P=0.03 ; N w$ vs $C f, P<0.001 ; C w$ vs $C f, P<0.001$

T3: Nw vs Nf, $\mathrm{P}<0.001$; Nw vs $\mathrm{Cw}, \mathrm{P}=0.012$; Nw vs $\mathrm{Cf}, \mathrm{P}<0.001$; $\mathrm{Cw}$ vs $\mathrm{Cf}, \mathrm{P}<0.001$

Water intake

T1: Nw vs Nf, $\mathrm{P}<0.001 ; \mathrm{Cw}$ vs $\mathrm{Cf}, \mathrm{P}<0.001 ; \mathrm{Nf}$ vs $\mathrm{Cw}, \mathrm{P}<0.001$

T2: Nw vs Nf, $\mathrm{P}<0.001 ; \mathrm{Cw}$ vs $\mathrm{Cf}, \mathrm{P}<0.001 ; \mathrm{Nf}$ vs $\mathrm{Cw}, \mathrm{P}<0.001$; Nw vs $\mathrm{Cf}, \mathrm{P}<0.001$

T3: Nw vs Nf, $\mathrm{P}<0.001$; Cw vs $C f, P<0.001$; Nf vs $C w, P<0.001$; Nw vs $C f, P<0.001$

Nw group - offspring of control mothers receiving tap water

Nf group - offspring of control mothers receiving high fructose water

$\mathrm{Cw}$ - offspring exposed to caffeine during gestation receiving tap water

Cf - offspring exposed to caffeine during gestation receiving high fructose water

No differences were observed in renal or hepatic tissue swelling between groups. However, hematoxylineosin stained renal tissue samples obtained from $\mathrm{Cw}$ and $\mathrm{Cf}$ groups revealed a statistically significant higher number of cases (50\% in group $\mathrm{Cw}$ and $70 \%$ in group $\mathrm{Cf}$ ) with oedema, compared to group $\mathrm{Nw}$ (Nw vs $\mathrm{Cw}$, $\mathrm{P}=0.033$ and Nw vs Cf, $\mathrm{P}=0.003$ ) (Table 3, Figure 2). 


\section{Cureus}

\begin{tabular}{|c|c|c|c|c|c|}
\hline \multirow[t]{2}{*}{ Lesion in renal or hepatic tissue } & & \multicolumn{4}{|c|}{ Number of animals per group presented as percentage (\%) } \\
\hline & & Nw (\%) & $\mathrm{Nf}(\%)$ & Cw (\%) & Cf (\%) \\
\hline Oedema in renal tissue & Presence & $0^{\mathrm{a}, \mathrm{b}}$ & 40 & $50^{\mathrm{a}}$ & $70^{b}$ \\
\hline Swelling in renal tissue & Presence & 30 & 10 & 50 & 30 \\
\hline Oedema in hepatic tissue & Presence & 0 & 0 & 10 & 10 \\
\hline Swelling in hepatic tissue & Presence & 0 & 0 & 0 & 0 \\
\hline
\end{tabular}

TABLE 3: Analysis of histopathologic examination of renal and hepatic tissue samples, in relation to the presence or absence of oedema or swelling

Figures sharing the same superscript letters differentiate significantly from each other.

Renal tissue oedema: $\mathrm{Nw}$ vs $\mathrm{Cw}, \mathrm{P}=0.033$ and $\mathrm{Nw}$ vs $\mathrm{Cf}, \mathrm{P}=0.003$

Nw group - offspring of control mothers receiving tap water

Nf group - offspring of control mothers receiving high fructose water

$\mathrm{Cw}$ - offspring exposed to caffeine during gestation receiving tap water

Cf - offspring exposed to caffeine during gestation receiving high fructose water



FIGURE 2: Haematoxylin/Eosin stains of renal tissues

A. Males born of control mothers which received tap water; B. Males born of control mothers, which received a high fructose water solution $(200 \mathrm{~g} / \mathrm{l})$ daily instead of tap water; C. Males prenatally exposed to caffeine which received tap water; $D$. Males prenatally exposed to caffeine which received a high fructose water solution $(200 \mathrm{~g} / \mathrm{l})$ daily instead of tap water. The arrow in Figure $C$ indicates hydropic degeneration of tubular epithelium. The encircled area in Figure $C$ and the whole area in Figure $D$ present tissue with interstitial oedema. (A, C: 100x, B, D: 50x)

\section{Discussion}

In our study, we observed elevated triglyceride levels in male rats prenatally exposed to caffeine and having 
received a high fructose diet in adulthood (group Cf) compared to all other the experimental groups, including group Nf (offspring of mothers not receiving caffeine but under high fructose diet). This observation directly implicates prenatal caffeine exposure in metabolic homeostasis during adulthood. Similar findings were identified in previous studies where rats prenatally were exposed to caffeine (120 $\mathrm{mg} / \mathrm{Kg}$ of body weight) and received a high-fat diet in adulthood [11, 20]. Both studies support the hypothesis that such a diet triggers excessive lipogenesis in rats prenatally exposed to caffeine, the latter appearing to be particularly susceptible to develop metabolic syndrome. One of the studies also supported that this effect eventually leads to hepatic macrovesicular steatosis [20]. This finding was not established in our study, perhaps due to the lower dose of caffeine administered during the gestational period (20 $\mathrm{mg} / \mathrm{kg} /$ day). Nevertheless, all studies, including ours, show clear metabolic deterioration following antenatal caffeine exposure during adulthood using two different metabolic syndrome models (high-fat diet and high fructose diet).

Food consumption was significantly lower in fructose groups $\mathrm{Nf}$ and $\mathrm{Cf}$, compared to control diet groups $\mathrm{Nw}$ and $\mathrm{Cw}$. This may be attributed to higher fluid consumption in the fructose groups, which, as previously observed, leads to an additional calorie intake via the fructose solution [19]. Furthermore, the sweet-tasting fructose enhanced the palatability of the solution and was thus preferred by the rats over the standard chow. Importantly, $\mathrm{Nw}$ and $\mathrm{Cw}$ (control diet groups), as well as $\mathrm{Nf}$ and $\mathrm{Cf}$ (fructose diet groups), had similar food and water consumption levels ( $\mathrm{Nw}$ vs $\mathrm{Cw}$, and $\mathrm{Nf}$ vs $\mathrm{Cf}$ ).

Studies in adult rats have shown favorable outcomes of caffeine administration in connection with insulin sensitivity, blood glucose, and hypertension [9]. In the study of Conte et al., $1 \mathrm{~g} / \mathrm{L}$ of caffeine was administered to adult rats via drinking water for 15 days. On the contrary, in our study, the administration of caffeine occurred during intrauterine life, possibly affecting organ development, as well as creating a different environment inducing epigenetic changes and offering different possible explanations for the differences observed.

In this study, prenatally caffeine exposure (PCE) in rats' offspring led also to raised systolic blood pressure compared to the control group. The difference was even more profound when combined with a high fructose diet. To our knowledge, this is the first study to indicate that maternal caffeine consumption may increase the susceptibility of male offspring to hypertension. However, the underlying mechanism remains so far undetermined, and therefore, further research is required.

A potential pathophysiologic pathway, possibly triggering this effect, appears to be that of renal developmental abnormalities caused by PCE, including glomerulosclerosis and interstitial sclerosis [21]. Glomerulosclerosis is a glomerular lesion that leads to a progressive deterioration in renal function. In the aforementioned study, histological examination of tissue samples harvested from adult offspring antenatally exposed to caffeine revealed pathological features and alterations in renal architecture. Structural damage was identified in podocytes, a group of highly differentiated cells responsible for the maintenance of the glomerular basement membrane, accompanied by a reduction in podocyte markers, most importantly that of renal angiotensin II receptor type 2 (AT2R) gene expression. PCE offspring kidneys exhibited an enlarged Bowman's space and significantly reduced glomerular tuft and cortex width, as well as an increased nephrogenic to cortical zone ratio. The authors proposed that the compromised AT2R functional programming observed in the PCE group may partially mediate the developmental glomerulosclerosis in adults, which in turn generates hypertension. Furthermore, the renin-angiotensin system (RAS) is crucial to kidney development. Therefore, any potential impairment in this system could lead to renal morphological abnormalities or dysfunction. A study in rats showed that a dose of $180 \mathrm{mg} / \mathrm{kg}$ per day of caffeine during pregnancy increased maternal plasma Angiotensin-II levels, leading to chronic activation of the maternal and placental RAS [22]. Other animal experiments suggested that prenatally developed renal dysplasia leads to high susceptibility to renal diseases [23-25]. In our study, both caffeine exposure groups (Cw and Cf) had higher frequencies of adult renal oedema ( $50 \%$ in group $\mathrm{Cw}$, and $70 \%$ in group $\mathrm{Cf}$ ) compared to the control groups.

We also observed higher systolic blood pressure in the caffeine exposure groups compared to $\mathrm{Nw}$ and $\mathrm{Nf}$ groups. Furthermore, we also observed hypertriglyceridemia in both caffeine groups compared to controls, showing multiple constituents of the metabolic syndrome being affected by antenatal caffeine exposure. It has been shown previously that caffeine consumption during pregnancy resulted in elevated levels of maternal glucocorticoids, which inhibit the activity of the fetal hypothalamic-pituitary-adrenal (HPA) axis via a negative feedback regulation [26]. This impairment led to intrauterine growth restriction (IUGR) and increased the risk of developing metabolic syndrome in adulthood. The findings in our study indicate a similar susceptibility in developing metabolic syndrome, as shown by the high levels of triglycerides and raised blood pressure.

Literature about human fetuses exposed to caffeine showed that children whose mothers consumed 4-5.9 and $\geqslant 6$ units of caffeine per day ( 1 unit=90 $\mathrm{mg}$ caffeine) during pregnancy tended to have a higher childhood body mass index and total body fat mass [27].

\section{Conclusions}


The results of our study clearly show phenotypic effects of PCE in the development of metabolic syndrome in adulthood. Certainly, as in all experimental studies, the results have to be viewed under a translational lens and not directly transferred to humans. Nevertheless, our study is the first to examine the effects of PCE in a model of adult metabolic syndrome induced by a high fructose diet. We observed significant interaction of PCE with the presence of lower birth weight, high blood pressure, renal oedema, and hypertriglyceridemia. Taking into account that caffeine doses used in our study were well within the accepted range for human pregnancies, as well as how widespread coffee consumption is during pregnancy, these findings at least provide significant motivation for further studies.

\section{Additional Information \\ Disclosures}

Human subjects: All authors have confirmed that this study did not involve human participants or tissue. Animal subjects: The experimental protocol was reviewed by the Bioethics Committee for studies using animals for scientific purposes and approved by the Veterinary Directorate of Attica Region, in accordance with the EU Directive 2010/63/EE. Issued protocol number 1264/11-05-2015 . Conflicts of interest: In compliance with the ICMJE uniform disclosure form, all authors declare the following: Payment/services info: All authors have declared that no financial support was received from any organization for the submitted work. Financial relationships: All authors have declared that they have no financial relationships at present or within the previous three years with any organizations that might have an interest in the submitted work. Other relationships: All authors have declared that there are no other relationships or activities that could appear to have influenced the submitted work.

\section{Acknowledgements}

We wish to thank Mrs. Esmeralda Ntousi, Mr. Panagiotis Tsakiropoulos, and Mr. Nikolaos Tsakiropoulos for their assistance in the experimental procedures.

\section{References}

1. Fulgoni VL, Keast DR, Lieberman HR: Trends in intake and sources of caffeine in the diets of US adults: 2001-2010. Am J Clin Nutr. 2015, 101:1081-7. 10.3945/ajcn.113.080077

2. Goldstein A, Warren R: Passage of caffeine into human gonadal and fetal tissue . Biochem Pharmacol. 1962, 1962:166-8. 10.1016/0006-2952(62)90106-5

3. Temple JL, Bernard C, Lipshultz SE, Czachor JD, Westphal JA, Mestre MA: The safety of ingested caffeine: a comprehensive review. Front Psychiatry. 2017, 8:80-80. 10.3389/fpsyt.2017.00080

4. American College of Obstetricians and Gynecologists: ACOG Committee Opinion No. 462: Moderate caffeine consumption during pregnancy. Obstet Gynecol. 2010, 1:467-8. 10.1097/AOG.0b013e3181eeb2a1

5. Wang S, Noh SK, Koo SI: Epigallocatechin gallate and caffeine differentially inhibit the intestinal absorption of cholesterol and fat in ovariectomized rats. J Nutr. 2006, 136:2791-6. 10.1093/jn/136.11.2791

6. Kobayashi-Hattori K, Mogi A, Mathumoto Y, Takita T: Effect of caffeine on the body fat and lipid metabolism of rats fed on a high-fat diet. Biosci Biotechnol Biochem. 2005, 69:2219-23. 10.1271/bbb.69.2219

7. Sugiura C, Nishimatsu S Moriyama T, Ozasa S, Kawada T, Sayama K: Catechins and caffeine inhibit fat accumulation in mice through the improvement of hepatic lipid metabolism. J Obes. 2012, 2012:520510. $10.1155 / 2012 / 520510$

8. Panchal SK, Wong WY, Kauter K, Ward LC, Brown L: Caffeine attenuates metabolic syndrome in dietinduced obese rats. Nutrition. 2012, 28:1055-62. 10.1016/j.nut.2012.02.013

9. Conde SV, Nunes da Silva T, Gonzalez C, Carmo MM, Monteiro E, Guarino MP: Chronic caffeine intake decreases circulating catecholamines and prevents diet-induced insulin resistance and hypertension in rats. Br J Nutr. 2012, $107: 86-95.10 .1017 /$ S0007114511002406

10. Kalankesh LR, Dastgiri S, Rafeey M, Rasouli N, Vahedi L: Minimum data set for cystic fibrosis registry: a case study in Iran. Acta Inform Med. 2015, 23:18-21. 10.5455/aim.2015.23.18-21

11. Li J, Luo H, Wu Y, et al.: Gender-specific increase in susceptibility to metabolic syndrome of offspring rats after prenatal caffeine exposure with post-weaning high-fat diet. Toxicol Appl Pharmacol. 2015, 284:345-53. 10.1016/j.taap.2015.03.002

12. Sun T, Guo J, Chen H, et al.: Maternal caffeine exposure impairs insulin secretion by pancreatic beta-cells and increases the risk of type II diabetes mellitus in offspring. Cell Biol Int. 2014, 38:1183-93. 10.1002/cbin.10321

13. Kou H, Wang G, Pei L: Effects of prenatal caffeine exposure on glucose homeostasis of adult offspring rats . Sci Nat. 2017, 104:89. 10.1007/s00114-017-1510-4

14. Guo Y, Luo H, Wu Y, Magdalou J, Chen L, Wang H: Influencing factors, underlying mechanism and interactions affecting hypercholesterolemia in adult offspring with caffeine exposure during pregnancy. Reprod Toxicol. 2018, 79:47-56. 10.1016/j.reprotox.2018.05.005

15. Luo H, Li J, Cao H, Tan Y, Magdalou J, Chen L, Wang H: Prenatal caffeine exposure induces a poor quality of articular cartilage in male adult offspring rats via cholesterol accumulation in cartilage. Sci Rep. 2015, 5:17746. 10.1038/srep17746

16. Bae J, Choi H, Choi Y, Roh J: Dose- and time-related effects of caffeine on the testis in immature male rats . Exp Anim. 2017, 66:29-39. 10.1538/expanim.16-0060

17. Mendelsohn ME, Karas RH: The protective effects of estrogen on the cardiovascular system . N Engl J Med. 1999, 340:1801-11. 10.1056/NEJM199906103402306

18. Palmisano BT, Zhu L, Stafford JM: Role of estrogens in the regulation of liver lipid metabolism . Adv Exp Med Biol. 2017, 2017:227-256. 10.1007/978-3-319-70178-3_12 
19. Mamikutty N, Thent ZC, Sapri SR, Sahruddin NN, Yusof MRM, Suhaimi FH: The establishment of metabolic syndrome model by induction of fructose drinking water in male Wistar rats. Biomed Res Int. 2014, 2014:263897. 10.1155/2014/263897

20. Wang L, Shen L, Ping J, et al.: Intrauterine metabolic programming alteration increased susceptibility to non-alcoholic adult fatty liver disease in prenatal caffeine-exposed rat offspring. Toxicol Lett. 2014, 224:311-8. 10.1016/j.toxlet.2013.11.006

21. Ao Y, Sun Z, Hu S, et al.: Low functional programming of renal AT2R mediates the developmental origin of glomerulosclerosis in adult offspring induced by prenatal caffeine exposure. Toxicol Appl Pharmacol. 2015, 287:128-138. 10.1016/j.taap.2015.05.007

22. Huang J, Zhou S, Ping J, et al.: Role of p53-dependent placental apoptosis in the reproductive and developmental toxicities of caffeine in rodents. Clin Exp Pharmacol Physiol. 2012, 39:357-63. 10.1111/j.1440-1681.2012.05676.x

23. Zimanyi MA, Denton KM, Forbes JM, Thallas-Bonke V, Thomas MC, Poon F, Black MJ: A developmental nephron deficit in rats is associated with increased susceptibility to a secondary renal injury due to advanced glycation end-products. Diabetologia. 2006, 49:801-10. 10.1007/s00125-006-0175-x

24. Plank C, Nüsken KD, Menendez-Castro C, et al.: Intrauterine growth restriction following ligation of the uterine arteries leads to more severe glomerulosclerosis after mesangioproliferative glomerulonephritis in the offspring. Am J Nephrol. 2010, 32:287-95. 10.1159/000319045

25. Boubred F, Laurent, Buffat C, et al.: Early postnatal overfeeding induces early chronic renal dysfunction in adult male rats. Am J Physiol Renal Physiol. 2009, 297:943-51. 10.1152/ajprenal.90704.2008

26. Xu D, Zhang B, Liang G: Caffeine-induced activated glucocorticoid metabolism in the hippocampus causes hypothalamic-pituitary-adrenal axis inhibition in fetal rats. PLoS One. 2012, 7:9. 10.1371/journal.pone.0044497

27. Voerman E, Jaddoe VWV, Gishti O, Hofman A, Franco OH, Gaillard R: Maternal caffeine intake during pregnancy, early growth, and body fat distribution at school age. Obesity (Silver Spring). 2016, 24:11701177. 10.1002/oby.21466 